\title{
Evaluación de prácticas silviculturales y su influencia en la composición florística en un claro vegetal ubicado en la Estación Ambiental de Tutunendo, Chocó, Colombia
}

\section{Assessment practices in forestry and their influence floristic composition light plant located in the Estación Ambiental de Tutunendo, Chocó, Colombia}

\section{Mirla Perea Murillo', Jovanny Mosquera Pino²}

\section{Resumen}

Con el objeto de determinar la composición florística, el crecimiento y desarrollo de especies forestales de interés especial emergentes en un claro vegetal, dentro de un área de $2.860 \mathrm{~m}^{2}$, se demarcaron 11 cuadrantes mediante una selección sistemática, cada cuadrante tuvo un área de $10 \times 10 \mathrm{~m}$ para individuos en estado latizal y $2 \times 2 \mathrm{~m}$ para estado brinzal y asi determinar la composición, uniformidad, riqueza, dominancia y frecuencia de las especies emergentes en el claro vegetal; también se evaluó la efectividad de técnicas silviculturales que promuevan el crecimiento y desarrollo de las plántulas, realizadas en una cronosecuencia de 3 años (2011, 2012 y 2013), a las especies forestales, que por sus características ecológicas, culturales y económicas son denominadas de interés especial, comparado con la composición florística del año 2010 a las cuales no se les aplicó ninguna técnica de promoción. Así, se logró identificar 373 individuos, distribuidos en 21 familias, con una riqueza de 29 especies y uniformidad de 82\% durante los tres años de monitoreo; en los años 1 y 2 se comparten 12 especies, el año 1 y 3 comparten 14 y el año 2 y 3 comparten 23 especies en común. Todo lo anterior indica que la eliminación de especies vegetales no deseadas, podas de formación y mantenimiento, $y$ raleos promueven el crecimiento y desarrollo de especies forestales de interés especial emergente en el claro vegetal establecido y monitoreado en la Estación Ambiental del Alto San Juan.

Palabras clave: Aprovechamiento forestal, Claro vegetal, Chocó, Silvicultura, Tutunendo.

\begin{abstract}
In order to determine the floristic composition, growth and development of forest species of special interest emerging in a vegetable course, within an area of $2.860 \mathrm{~m}^{2} 11$ quadrants were demarcated by a systematic selection, each quadrant with an area of $10 \times 10 \mathrm{~m}$ for individuals latizal state and $2 \times 2 \mathrm{~m}$ for individuals in brinzal state and thus determine this composition, uniformity, wealth, dominance and frequency of emerging plant species in the clear; as well as evaluate the effectiveness of silvicultural techniques that promote the growth and development of seedlings, conducted in a chronosequence 3 years (2011, 2012 and 2013), forestry species, their ecological, cultural and economic characteristics are called of particular interest, compared to the floristic composition of the year 2010 which were not applied any
\end{abstract}

Ingeniera Agroforestal, Investigadora asociada tipo I del Instituto de Investigaciones Ambientales del Pacífico (IIAP), Quibdó, Chocó.

e-mail: mperea@iiap.org.co

2 Ingeniero Agroforestal, Investigador asociado tipo II del Instituto de Investigaciones Ambientales del Pacífico (IIAP), Quibdó, Chocó.

e-mail: jmosquera@iiap.org.co

Recibido: 10 de diciembre de 2013

Aprobado: 1 de marzo de 2014 


\section{Prácticas silviculturales y composición florística. M Perea, J Mosquera}

promotional technique. In this vein, it was possible to identify 373 individuals, distributed in 21 families, with a richness of 29 species and uniformity of $82 \%$ during the three years of monitoring; in the same way, in years 1 and 212 species are shared, year 1 and 3 share 14 species and year 2 and 3 share 23 species in common. All this indicates that the removal of plant species unwanted pruning training and maintenance, and thinning promote growth and development of forest species of special interest emerging in the plant clearly established and monitored in environmental station Alto San Juan.

Keywords: Clear vegetable, Chocó, Forestry, Tutunendo.

\section{Introducción}

En Colombia, el departamento del Chocó representa apenas un sexto del área del país, pero ha proveído hasta $55 \%$ de la madera extraída en los últimos 15 años (Fundación Natura et al. 2005). Según estadísticas del IIAP (2001), por cada $22 \mathrm{~m}^{3}$ de madera que se extraen del bosque en la región pacífica, se altera una hectárea de bosque; por las características de selectividad de especies que tienen los aprovechamientos persistentes, estos han generado un agotamiento de las especies de interés especial y que no son sometidas a un plan de manejo real; asimismo esta situación es una alerta sobre la necesidad de conservar la gran diversidad biológica presente en esta región del país. Partiendo de este análisis, el corregimiento de Tutunendo es reconocido por tener bosques donde la combinación de temperaturas cálidas y mucha lluvia contribuyen a un medio ambiente donde el crecimiento y la reproducción de las plantas es muy rápido, además se caracteriza por el aprovechamiento selectivo de madera, actividad que ha sido realizada ancestralmente por las familias que han subsistido gracias a esta actividad sin tecnificación alguna, dejando grandes áreas de tierra desprovistas de vegetación, las cuales se conocen como "claros", que también pueden ser provocados por acción de la naturaleza como lluvias y vientos fuertes provocando la caída de árboles de gran porte. Estos han sido definidos por Witmore (1989), Foster y Brokaw (1992), Runkle (1992), como un "hueco" vertical en el bosque, los cuales tienen un área de más de $20 \mathrm{~m}^{2}$ como consecuencia de disturbios naturales muy frecuentes que alteran la cobertura del dosel y tienen implicaciones sobre la dinámica de los bosques.

El estudio de la dinámica de claros juega un papel fundamental en la diversidad de los bosques tropicales, pues ayuda a comprender la composición, distribución, abundancia y características de la historia de vida de las especies vegetales (Arriaga 1988). Sumado a ello, en el presente documento se destaca la importancia de algunas especies con alto valor de uso que emergieron con la apertura del claro; estas especies presentan características especiales con fines forestales, teniendo en cuenta algunos criterios como la situación actual y aceptación de cada una de ellas en las comunidades que hacen parte del área de influencia de la Estación Ambiental de Tutunendo, además, se destaca el desarrollo y adaptación in situ, teniendo en cuenta algunas prácticas agrosilvícolas para mejorar su crecimiento, desarrollo, promoción y conservación, porque son de gran interés por sus usos.

Este artículo pretende aportar elementos valiosos sobre el manejo de la vegetación remanente en claros formados por el aprovechamiento selectivo de madera, evaluando prácticas silviculturales y su influencia en la composición florística en un claro vegetal, lo que contribuirá en el aporte de herramientas para el manejo de especies forestales en bosques perturbados por acciones antrópicas, de tal manera que se garantice la existencia de especies forestales de interés especial en los claros.

\section{Materiales y métodos}

Area de estudio. El claro se encuentra ubicado dentro del área operativa de la Estación Ambiental de Tutunendo, en el corregimiento de Tutunendo, municipio de Quibdó, a $5^{\circ} 45^{\prime} 43.1$ " norte y a $76^{\circ} 31 ' 21.2^{\prime \prime}$ oeste; se encuentra a 150 metros sobre el nivel de mar, presenta una temperatura megatermal $\left(25.7\right.$ a $\left.27.9^{\circ} \mathrm{C}\right)$ y una precipitación promedio anual de $10.000 \mathrm{~mm}$ (Figura 1) (Poveda et al. 2004, citado por Ramírez y Galeano 2011).

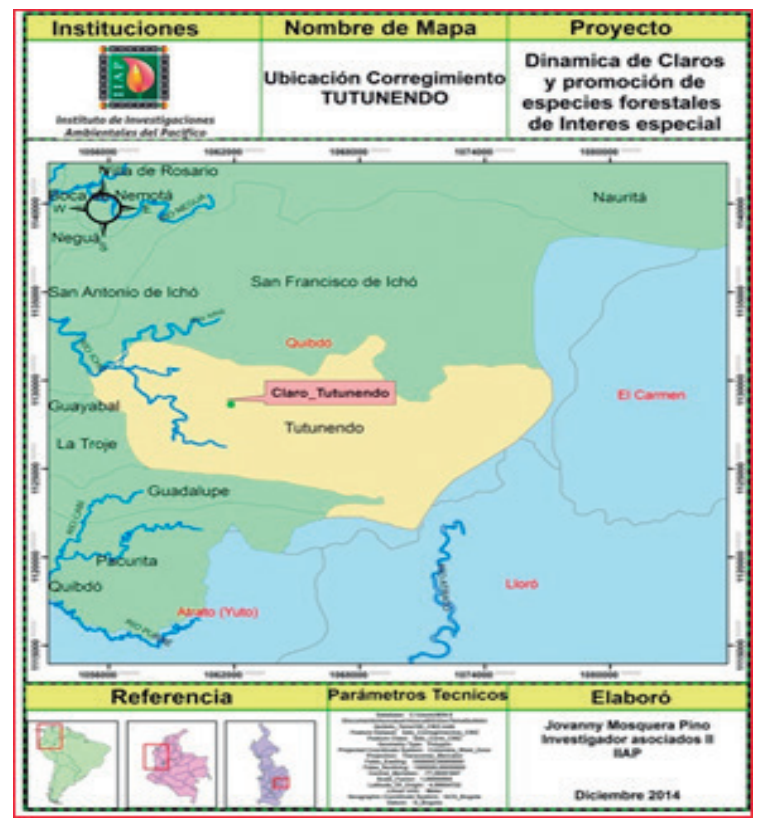

Figura 1. Localización del claro. 


\section{Bioetnia Volumen 11, 2014}

\section{Métodos}

Criterios para la selección de especies de interés especial. Para la selección de las especies forestales con alto valor comercial y que se encuentran en estado de regeneración se tuvo en cuenta los siguientes criterios:

Buena aceptación de las especies forestales por la comunidad y sobre todo por los aserradores de la región que dan fe del éxito del producto en el mercado.

Que la escogencia de los individuos para el estudio correspondieran a especies de buena calidad y que alcancen un alto precio en cuanto a las ventas.

- Que la utilización de las especies forestales sea para diversos usos en la construcción y transformación de la madera.

Delimitación y monitoreo del claro. Para la identificación de las especies forestales de interés especial se demarcaron 11 cuadrantes mediante selección sistemática; cada cuadrante con un área de $10 \times 10 \mathrm{~m}$ para individuos en estado latizal y 2 × $2 \mathrm{~m}$ para individuos en estado brinzal. Lo anterior permite cuantificar y hacer seguimiento a la regeneración natural de las especies con capacidad de formar parte del estrato superior del bosque en un futuro y cerrar el claro.

Seguimiento. El monitoreo de las especies forestales de interés especial, se realizó con una frecuencia de toma y registro de datos cada 30 días; para ello, se elaboró un formato en el que se tuvo en cuenta datos cuantitativos y cualitativos como nombre de las especies, número de la parcela o cuadrante y sus respectivas coordenadas, cantidad de individuos en pie, DAP y altura.

Diseño y aplicación de estrategias silvícolas. En conversatorios con actores sociales claves, se aplicaron instrumentos etnicosociales para el registro de información necesaria relacionada con técnicas silviculturales que garantizaron la promoción de especies vegetales de interés especial; para ello, apoyados en lluvias de ideas, se listaron y se seleccionaron las estrategias de raleo, podas de formación y mantenimiento y eliminación de arvenses.

Determinación de la estructura horizontal. Se utilizó un modelo lineal con análisis en series de tiempo para relacionar entre si y ordenar las especies con base en sus características por sitios de muestreos, también se calcularon los índices ecológicos correspondiente a la composición florística (abundancia, frecuencia, dominancia, IVI, riqueza y uniformidad) y Jaccard utilizando el programa SIMIL y DIVERS.

\section{Resultados y discusión}

Se establecieron 11 cuadrantes seleccionados de forma sistemática dentro del claro en estudio en un área de 2.860 $\mathrm{m}^{2}$, registrando un total de 373 individuos, perteneciente a 29 especies distribuidas en 21 familias, durante los 3 años de investigación (2011 a 2013); en el año 2011 se inició con una riqueza específica de 16 especies, aumentando en 24 especies para el año 2012, hasta llegar a 29 especies en el 2013; de esta manera, Virola sebifera Aubl con 80 individuos registró la mayor cantidad equivalente al $41 \%$, seguido de Carapa guianensis con (17\%), Lumania parviflora, Adenaria floribunda y Wettinia quinaria con $14 \%$ cada una. Las especies menos abundantes fueron Pouteria sp, Guatteria cargadero Triana, Dipterix oleifera Benth, Inga marginata Willd y Zanthoxylum tachuelo.

De las 21 familias registradas, la más abundante fue Mimosacea con 4 (14\%) especies, seguida de Moraceae, Tiliaceae, Sapotaceae, Burseraceae y Bombacaceae con 2 (7\%) especies cada una.

En relación con la frecuencia, Protium veneralense, Enterolobium ciclocarpum, Michopholis guyanensis, Guatteria aff. calimensis, Perebea guianensi, Hevea brasiliensis, Lumania parviflora, Ocotea cernua, Virola cuspidata, Grias cauliflora y Zanthoxylum tachuelo con $4.5 \%$ fueron las especies más frecuentes, lo cual quiere decir que se registraron en cada año de monitoreo.

En lo que a dominancia se refiere, la especie que predominó en esta categoría fue el nuánamo, seguida de Tapirira meryantha, Lumania parviflora, Adenaria floribunda y Wettinia quinaria; las menos dominantes fueron Pouteria sp, Guatteria aff. calimensis, Dipterix oleifera Benth, Inga marginata Willd y Coraipa sp.

Es de resaltar que la dominancia de especies está estrechamente ligada con las prácticas silviculturales utilizadas en el claro, lo cual demuestra la diversidad de las mismas y la importancia de realizar o no este tipo de prácticas para mejorar el crecimiento y desarrollo de los individuos. Esta teoría de utilización de manejo silvicultural coincide con Wadsworth y Zweede (2006, citados por Guariguata et al. 2012), quienes argumentan que la práctica de raleos de liberación, se hace con el fin de eliminar árboles vecinos para estimular el crecimiento de árboles maderables para un futuro, pero es contrario a lo que dice Sheil et al. (2008), quienes están en desacuerdo con que se hagan raleos, porque las plantas alimenticias, medicinales y palmas que son cortadas en bosques tropicales, pueden tener múltiple usos.

El índice de similitud de Jaccard indica que no existen disimilitudes entre los años, lo cual significa que los 3 años comparten al menos una especie emergente en los claros. A su vez, el año 3 presenta similitud del $88 \%$ de especies contrario a los años 1 y 2 que no alcanzan el $50 \%$ de similitud (Tabla 1).

La uniformidad es de 0,8 , lo cual quiere decir, que de las 29 especies identificadas en el claro durante los 3 años al menos $82 \%$ de estas se encuentran presentes en cada 


\section{Prácticas silviculturales y composición florística. M Perea, J Mosquera}

Tabla 1

Indice de similaridad

\begin{tabular}{ccccc}
\hline Años de estudio & Sp. comunes & Jaccard & Sorenson & Morisita \\
\hline $1-3$ & 14 & 0.483 & 0.651 & 0.723 \\
$1-2$ & 12 & 0.429 & 0.600 & 0.686 \\
$2-3$ & 23 & 0.885 & 0.939 & 0.864 \\
\hline
\end{tabular}

año de muestreo.

Considerando grande el claro estudiado, se esperaba encontrar más individuos de Cecropiaceae y Melastomataceae, entre otras, y únicamente se registra Cespedecia macrophila que es una heliófita durable (Palacios 2004). Lo anterior ha sido visto por Foster y Brokaw (1992) en Barro Colorado, Panamá y donde manifiestan que es muy común que los claros menores de $150 \mathrm{~m}^{2}$, sean colonizados principalmente por especies persistentes que pueden sobrevivir varios años en la sombra, a diferencia de claros más grandes donde solo crecen otras especies denominadas pioneras (o colonizadoras), que parecen necesitar de un claro continuo para lograr la madurez.

\section{Conclusión}

En los claros muestreados en el año 2010, se identificaron 27 especies pertenecientes a 15 familias, muchas de ellas habitantes del dosel en el bosque o esciófitas parciales, por lo que se puede pensar que no durarían mucho en el claro, porque están adaptadas para el rápido crecimiento, pero no para una vida larga dentro del bosque, lo cual es característico de las especies pioneras en claros de bosques tropicales. Contrario a los resultados obtenidos por la aplicación de las prácticas silviculturales, que mediante su implementación en los individuos presentes en el claro, permitió el desarrollo de 29 especies en 21 familias que garantizaron la presencia de las mismas y pasar de un estadío de brinzal a latizal especies como cargadero negro, cedro macho, chucho nuevo, carrá, choibá, coralito, corcho, guamo rosario, guayabo, hormigo, indio, jigua negro, pacó, para siempre, pastrán y peine mono y que no se registraron en el año 2010 por la alta tasa de reclutamiento al no aplicar dichas técnicas.

Por otro lado, la presencia de 29 especies y 21 familias parece ser un dato muy alentador y muestra la alta diversidad de este tipo de bosque. Así y a través de la ejecución de las prácticas silvícolas, se contribuyó a la eliminación de las plantas circundantes a las especies en estudio que competían por luz, agua y nutrientes; además fue una forma de estimularlas, evitando el ataque de plagas que pudieran ocasionar enfermedades para obtener un buen crecimiento y desarrollo, teniendo como resultado un total de 195 plantas en buen estado y solo 2 plantas muertas. Sin embargo, cabe destacar que las semillas de las especies pioneras no tienen mayores probabilidades de caer en claros del dosel que cualquier otro lugar, por consiguiente, puede ser posible que las pioneras más comunes en los claros estén representadas por adultos abundantes en el bosque o sean particularmente abundantes en bancos de semillas. También estaría preliminarmente demostrado, que los claros generados por el aprovechamiento selectivo de madera, en lugar de ser factores negativos, ayudan al mantenimiento de la diversidad.

\section{Literatura citada}

Arriaga L. 1988. Gap dynamics of a tropical cloud forest northeastern Mexico. Biotropica. 20: 178-84.

Foster R, Brokaw NV. 1992. Estructura e historia de la vegetación de la isla de Barro Colorado. En: Leigh E, Rand AE, Windsor DM (eds.). Ecología de un bosque tropical. Ciclos estacionales y cambios a largo plazo. Balboa: Smithsonian Tropical Research Institute; pp. 113-27.

Fundación Natura, Ministerio del Medio Ambiente, Universidad Nacional y Universidad de Antioquia. 2005. La ecorregión del Chocó Biogeográfico. Bogotá: Fundación Natura.

Guariguata MR, Locatelli B,Haupt F. 2012. Adapting tropical production forests to global climate change: risk perceptions and actions. Internat Forestry Rev. 14 (1): 27-38.

Instituto de Investigaciones Ambientales del Pacífico (IIAP) 2001. La minga. Caracterización rápida. Informe primera salida de campo. Estación Ambiental de Tutunendo. Quibdó, Chocó. p. 47.

Palacios W. 2004. Estrategias biológicas de las especies forestales: Los gremios forestales en los bosques tropicales húmedos del Ecuador. Lyonia. 1:33-40.

Ramírez G, Galeano G. 2011. Estudio de las comunidades de palma en dos regiones fitogeográfica del Chocó-Colombia. Maestría en Ciencias-Biología. Línea Biodiversidad y Conservación, Chocó-Colombia. Universidad Nacional de Colombia, Bogotá.

Runkle, J. 1992. Guidelines and sample protocol for sampling forest gaps. Gen. Tech. Rep. PNW-GTR-283. Portland: US Department of Agriculture, Forest Service, Pacific Northwest Research Station. 44 pp.

Sheil D, van Heist M, Liswanti N, Basuki I, Wan M. 2008. Biodiversity, landscapes and livelihoods: a local perspective. In: Moeliono M, Wollenberg $\mathrm{E}$, Limberg G (eds.). The decentralization of forest governance: politics, economics and the fight for control of forest in Indonesian Borneo. Londres: Earthscan; pp. 61-90.

Witmore T. 1989. An introduction to tropical rainforests. New York: Oxford University Press; 226 pp. 